\title{
Retrovirus-mediated transduction of a short hairpin RNA gene for GRP78 fails to downregulate GRP78 expression but leads to cisplatin sensitization in HeLa cells
}

\author{
TOSHIKAZU SUZUKI ${ }^{1,2}$, JUN LU $^{1}$, GAOFENG HU$^{1}$, KAZUKO KITA $^{1}$ and NOBUO SUZUKI ${ }^{1}$ \\ ${ }^{1}$ Department of Environmental Biochemistry, Graduate School of Medicine, Chiba University, \\ 1-8-1 Inohana, Chuo-ku, Chiba 260-8670; ${ }^{2}$ Department of Health and Nutrition, \\ Wayo Women's University, 2-3-1 Konodai, Ichikawa, Chiba 272-8533, Japan
}

Received October 25, 2010; Accepted December 16, 2010

DOI: $10.3892 /$ or.2011.1134

\begin{abstract}
Glucose-regulated protein 78 (GRP78) is expressed abundantly in various types of cancer cells and is believed to contribute to chemotherapeutic resistance. In this study, we investigated the effect of a continuous approach for the expression of a short hairpin RNA (shRNA) targeted to GRP78 with retrovirus transduction on the sensitivity to the anticancer drugs VP-16 and cisplatin. The reduction of GRP78 expression failed, and the expression of GRP94 and P5 chaperon mRNA increased; this increase was associated with a mild activation of the unfolded protein response in HeLa cells, which were stably transduced with GRP78 shRNA gene. The transduced cells exhibited similar sensitivity to VP-16-induced cell death when compared to control GFP shRNA gene-transduced cells. However, sensitivity to cisplatin-induced cell death was higher in GRP78 shRNA gene-transduced cells compared to control cells. These results demonstrate that the continuous or prolonged approach targeting GRP78 confers sensitization of HeLa cells to cisplatin independently of the down-regulation of GRP78 expression. The role of the unfolded protein response in sensitization to cisplatin is discussed.
\end{abstract}

\section{Introduction}

Cancer cells in poorly vascularized solid tumors are often subjected to stressful microenvironments, including hypoxia, low $\mathrm{pH}$, and the deprivation of glucose and other nutrients

Correspondence to: Dr Toshikazu Suzuki, Department of Health and Nutrition, Wayo Women's University, 2-3-1 Konodai, Ichikawa, Chiba 272-8533, Japan

E-mail: t-suzuki@wayo.ac.jp

Dr Nobuo Suzuki, Department of Environmental Biochemistry, Graduate School of Medicine, Chiba University, 1-8-1 Inohana, Chuo-ku, Chiba 260-8670, Japan

E-mail:nobuo@faculty.chiba-u.jp

Key words: glucose-regulated protein 78, short hairpin RNA, the unfolded protein response, cisplatin sensitivity
(1). These microenvironments disrupt protein folding in the endoplasmic reticulum (ER) $(2,3)$. The accumulation of unfolded proteins in the ER activates the unfolded protein response (UPR) pathways and enhances the expression of ER chaperone proteins, including glucose-regulated protein (GRP) 78 and GRP94 (4-6). There are a number of studies stating that overexpression of GRP78 and GRP94 frequently occurs in various types of cancer, including prostate, breast, lung, esophageal, gastric, colon, brain and liver cancers (7-14).

GRP78 associates with nascent proteins, facilitates protein folding, prevents intermediates from aggregating, and targets misfolded proteins for proteasome degradation (15). GRP94 is thought to facilitate the assembly or oligomerization of folding intermediates in the ER (16). Both GRP78 and GRP94 also bind $\mathrm{Ca}^{2+}$, help to immobilize $\mathrm{Ca}^{2+}$, and maintain ER calcium homeostasis (4). With these properties, induction of GRP78 and GRP94 is involved in the protection of tumor cells from death under stressful microenvironmental conditions. Furthermore, overexpression of GRP78 and 94 may confer chemotherapeutic resistance to solid tumors $(5,17)$. Knockdown of GRP78 or GRP94 expression has been shown to sensitize cancer cells to chemotherapeutic agents and ionizing radiation $(13,18-23)$. Additionally, tumor cells derived from GRP78 heterozygous mice grew more slowly and showed enhanced apoptosis compared with tumor cells from GRP78 wild-type mice (24). Therefore, targeting GRP78/GRP94 expression or activity may be a promising anticancer strategy.

There is a possibility that the reduction of GRP78 expression increases the amount of misfolded or aggregated proteins, activates the UPR signaling pathway and enhances the expression of UPR-induced genes, including ER chaperone proteins, because GRP78 itself serves as a negative modulator of the UPR signaling pathways by binding to three ER stress sensor proteins: activating transcription factor 6 , inositolrequiring 1 protein (Ire1p) and protein kinase RNA-activatedlike ER kinase (PERK) (25).

We previously observed enhanced expression of GRP94, ER-localized DnaJ homologue 4 (ERdj4) and P5 chaperone mRNA, as well as activation of the GRP78 promoter, when the expression of GRP78 was suppressed by a small interfering RNA (siRNA) in HeLa cells (26). This finding suggests that the approach of prolonging suppression of GRP78 expression 
may fail to sensitize cells to anticancer drugs. On the contrary, it might confer chemotherapeutic resistance. To search for the possibility that such unintended consequences might occur under GRP78-targeted anticancer therapy, we used a retrovirus vector containing an shRNA-expressing gene targeted to GRP78. Transduction of an shRNA gene enabled us to suppress the expression of the target gene specifically and stably $(27,28)$. Retrovirus-mediated transduction is widely used to introduce genetic material efficiently and stably into the genome of any dividing cell type (29). HeLa cells were infected with the GRP78 shRNA vector and the stably transduced cells were selected. We then analyzed the expression of the UPR-activated genes, including GRP78 and GRP94, and sensitivity to two representative anticancer drugs, VP-16 and cisplatin, in the stable GRP78 shRNA gene-transduced cells.

\section{Materials and methods}

Plasmid construction and siRNA. The retrovirus vector pSUPER.retro (27), containing the H1-RNA promoter, was obtained from OligoEngine (Seattle, WA, USA). The pSUPER.retro vector was digested with BglII and HindIII and the following pairs of annealed oligos were ligated into the vector to create pSUPER.retro-shGRP78, pSUPER.retroshGRP94 and pSUPER.retro-shGFP, respectively: 5'-gat ccc ccA GTG TTG GAA GAT TCT GAT ttc aag aga ATC AGA ATC TTC CAA CAC Ttt ttt gga aa-3' and 5'-agc ttt tcc aaa aAA GTG TTG GAA GAT TCT GAT TCT GAT Ttc tct tga aAT CAG AAT CTT CCA ACA CTg gg-3'; 5'-gat ccc ccG AAG AAG CAT CTG ATT ACC ttc aag aga GGT AAT CAG ATG CTT CTT Ctt ttt gga aa-3' and 5'-agc ttt tcc aaa aaG AAG AAG CAT CTG ATT ACC tet ctt gaa GGT AAT CAG ATG CTT CTT Cgg g-3'; and 5'-gat ccc ccG CAA GCT GAC CCT GAA GTT Ctt caa gag aGA ACT TCA GGG TCA GCT TGC ttt ttg gaa a-3' and 5'-agc ttt tcc aaa aaG CAA GCT GAC CCT GAA GTT Ctc tct tga aGA ACT TCA GGG TCA GCT TGC ggg-3'. The 19 nt GRP78 and GRP94 target sequences and the 20 nt GFP target sequences are indicated in capitals in the oligonucleotide sequences. The target sequences for GRP78 and GRP94 were reported previously (26). The predicted sequences of the short hairpin transcripts of GRP78 shRNA, GRP94 shRNA and GFP shRNA are shown in Fig. 1A.

Adenovirus vector constructs were generated by excising shRNA-expressing cassette fragments from the retrovirus vectors with EcoRI and HindIII and blunt-ended with Klenow enzyme. These fragments were ligated into the BamHIand $\mathrm{XbaI}$-digested, and blunt-ended pENTR-U6 entry vector (Invitrogen, Carlsbad, CA, USA), yielding pENTR-shGRP78, pENTR-shGRP94 and pENTR-shGFP. Each shRNAexpressing cassette sequence in the pENTR vectors was transferred to an adenovirus vector, $\mathrm{pAd} / \mathrm{BLOCK}-\mathrm{iT}^{\mathrm{TM}}$-DEST, using Gateway ${ }^{\circledR}$ LR Clonase ${ }^{\mathrm{TM}}$ Enzyme mix (Invitrogen), yielding pAd/shGRP78, pAd/shGRP94 and pAd/shGFP.

Cell culture, transfection and viral infection. The cervical tumor cell line, HeLa S3, was obtained from the Japanese Collection of Research Bioresources Cell Bank (Osaka, Japan). The packaging cells for adenovirus production, 293A, were purchased from Invitrogen. Phoenix-Ampho packaging cells for retrovirus production (ATCC catalogue number SD 3443) were obtained from the American Type Culture Collection (Manassas, VA, USA). HeLa cells were cultured in Eagle's minimum essential medium (MEM; Nissui Pharmaceutical, Tokyo, Japan) containing 10\% (v/v) calf serum (Invitrogen). Phoenix and 293A packaging cells were maintained in Dulbecco's modified Eagle's medium (DMEM; Nissui Pharmaceutical) supplemented with $0.1 \mathrm{mM}$ MEM nonessential amino acids (Invitrogen), $60 \mu \mathrm{g} / \mathrm{ml}$ kanamycin (Sigma, St. Louis, MO, USA) and 10\% (v/v) fetal bovine serum (Roche Diagnostics, Mannheim, Germany). All cell lines were grown at $37^{\circ} \mathrm{C}$ in a humidified atmosphere containing $5 \% \mathrm{CO}_{2}$ and subcultured when confluence was reached.

Amphotropic retroviral supernatants were produced by transfection of each of the pSUPER.retro shRNA-expressing vectors into Phoenix-Ampho packaging cells using Lipofectamine $^{\mathrm{TM}} 2000$ reagent (Invitrogen). At $24 \mathrm{~h}$ posttransfection, the culture medium was refreshed, and the cells were incubated for a further $24 \mathrm{~h}$. The culture medium was then filtered through a $0.45 \mu \mathrm{m}$ filter, and each viral supernatant (Ret/shGRP78, Ret/shGRP94 and Ret/GFP) was used for infection of cells with polybrene solution $(4 \mathrm{mg} / \mathrm{ml})$. HeLa cells were infected for $5 \mathrm{~h}$ and allowed to recover for $48 \mathrm{~h}$ in fresh medium. The infected cells were cultured in the presence of $0.8 \mu \mathrm{g} / \mathrm{ml}$ puromycin for 8 days to remove uninfected cells. The stably infected cells were then used in experiments.

High titer adenoviral stocks ranging from $2-5 \times 10^{8} \mathrm{pfu} / \mathrm{ml}$ were produced by transfection of each of the pAd/shRNAexpressing vectors into 293A packaging cells according to the manufacturer's instructions for the BLOCK-iT Adenoviral RNAi Expression System (Invitrogen). HeLa cells were infected with the each of the adenoviral stocks (Ad/shGRP78, Ad/shGRP94 and Ad/shGFP) at an MOI of 50 for $24 \mathrm{~h}$. The infected cells were replaced with fresh medium, cultured for a further $24 \mathrm{~h}$ and used for Western blot analysis.

Western blot analysis. Western blot analysis was carried out as described previously (26). Whole cell protein samples were separated on $10 \%$ SDS-polyacrylamide gels and transferred to polyvinylidene difluoride membranes (Millipore, Bedford, MA, USA). Detection of GRP78, GRP94, the cleaved form of caspase-3 and $B$-actin proteins was performed with mouse anti-GRP78 (Assay Design, Ann Arbor, MI, USA), rat antiGRP94 (Assay Design), rabbit anti-cleaved caspase-3 (Trevigen, Gaithersburg, MD, USA) and mouse anti- $\beta$-actin (MP Biomedicals Inc., Aurora, OH, USA) monoclonal antibodies, respectively. The antigen-antibody complexes were detected by horseradish peroxidase-conjugated anti-mouse IgG (GE Healthcare, Buckinghamshire, UK), anti-rabbit IgG (GE Healthcare) or anti-rat IgG (Assay Design) and visualized using the ECL system (GE Healthcare) according to the manufacturer's instructions.

$R T-P C R$. Isolation of total RNA, first-strand cDNA synthesis and RT-PCR analysis was carried out as described previously (26). The following sets of primers were used: 5'-GTT CTT CAA TGG CAA GGA ACC ATC-3' and 5'-CCA TCC TTT 
A

$\begin{array}{ll}\text { GRP78 shRNA } & 5^{\prime}-\text { agu guu gga aga uuc uga uu } \mathrm{uu}_{\mathrm{a}} \mathrm{c}_{\mathrm{a}}-\text { uu uca caa ccu ucu aag acu } \mathrm{aag}_{\mathrm{a}} \mathrm{g}^{\mathrm{a}}\end{array}$

GRP94 shRNA 5'- gaa gaa gca ucu gau uac $\mathrm{cu}^{\mathrm{u}} \mathrm{c}_{\mathrm{a}}$

3'- uu cuu cuu cgu aga cua aug ga $_{\mathrm{g}_{\mathrm{a}}} \mathrm{g}^{\mathrm{a}}$

GFP shRNA $\quad 5^{\prime}-\quad$ gca agc uga ccc uga agu ucu ${ }^{\mathrm{u}} \mathrm{c}_{\mathrm{a}}$

3'- uu cgu ucg acu ggg acu uca aga $_{\mathrm{g}_{\mathrm{a}}} \mathrm{g}^{\mathrm{a}}$

B

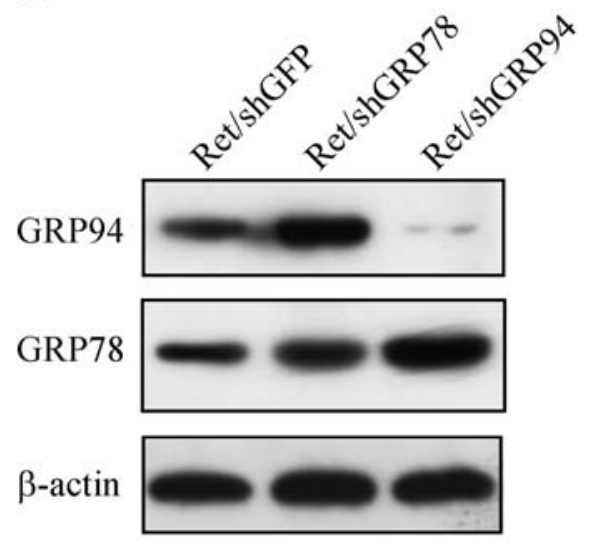

C

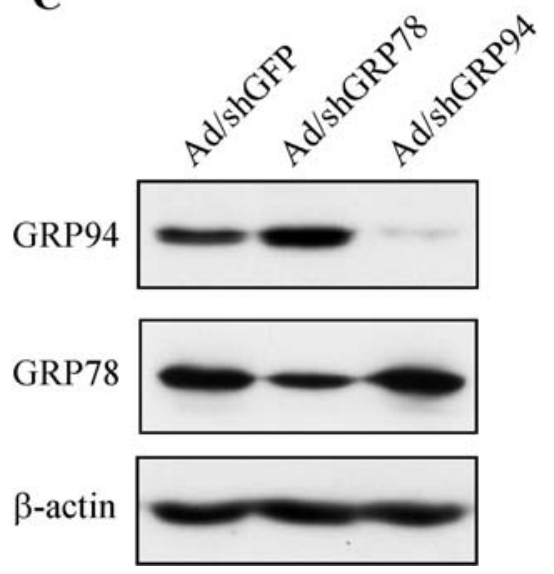

Figure 1. Effect of GRP78 shRNA gene transduction on GRP78 and GRP94 protein levels in HeLa cells. (A) Sequences of the predicted shRNA specific to GRP78, GRP94 and GFP (control) mRNA. (B) Stable transduction of the GRP78 shRNA gene by a retrovirus vector failed to suppress the expression of GRP78 protein but increased the expression of the GRP94 protein. Cell lysates prepared from the surviving population of the transduced cells were analyzed by Western blot analysis. The amount of actin was used as a loading control. Ret/shGFP, Ret/shGRP78, and Ret/shGRP94 represent the retrovirus vectortransduced cells, respectively. (C) Transient expression of GRP78 shRNA with an adenovirus vector suppressed the expression of GRP78 protein and increased the expression of GRP94. Cell lysates prepared 2 days after infection with adenovirus were analyzed by Western blot analysis. Ad/shGFP, Ad/shGRP78, and Ad/ shGRP94 represent the adenovirus vector-infected cells, respectively.

CGA TTT CTT CAG GTG-3' for GRP78; 5'-AAG AAC CTG CTG CAT GTC ACA GAC-3' and 5'-ATG GGC TCC TCA ACA GTT TCA GTC-3' for GRP94; 5'-TTC CAG ACT GAT CCA ACT GCA GAG-3' and 5'-AGC TGT GCC ACT TTC CTT TCA TTC-3' for CHOP; 5'-TCG GAG GGT GCA GGA TAT TAG AAA-3' and 5'-GGA TCC AGT GTT TTG GTT TTG ACC-3' for ERdj4; 5'-GAA AGG CGA GTC TCC TGT GGA TTA-3' and 5'-AGA GCT CCC TGA GAA ACT CGT TGA-3' for P5; 5'-GAC CTC AAC TAC ATG GTC TAC ATG-3' and 5'-TGT CGC TGT TGA AGT CAG AGG AGA C-3' for glyceraldehyde-3-phosphate dehydrogenase (GAPDH); and 5'-CCT TGT AGT TGA GAA CCA GG-3' and 5'-GGG GCT TGG TAT ATA TGT GG-3' for $\mathrm{X}$-box binding protein $1(X B P 1)$. The PCR products were visualized after electrophoresis on $1.5 \%(\mathrm{w} / \mathrm{v})$ agarose gels by ethidium bromide staining. To detect the spliced form of $X B P 1$ cDNA, samples were electrophoresed on $4 \%$ polyacrylamide gels to separate the spliced form from the unspliced form.

Determination of drug sensitivity. Cell viability after treatment with VP-16 or cisplatin was determined by a colony-formation assay. Cells were seeded onto $60-\mathrm{mm}$ dishes at a density of 200-300 cells per dish. The next day,
VP-16 $(0-40 \mu \mathrm{M})$ or cisplatin $(0-20 \mu \mathrm{M})$ were added to the cell cultures. One hour after incubation, drug-containing media were replaced with fresh MEM containing $10 \%$ calf serum, and the cells were cultured for 2 weeks to form colonies. Colonies comprising $>50$ cells were counted after staining with $0.1 \%(\mathrm{w} / \mathrm{v})$ methylene blue tetrahydrate. The survival fraction of each drug concentration was calculated as the ratio of colony numbers in the drug-treated samples to those in mock-treated samples.

\section{Results}

Expression of GRP78 is not suppressed in GRP78 shRNA gene-transduced HeLa cells. We isolated HeLa cells transduced with the GRP78 shRNA gene by retrovirus infection with Ret/shGRP78. We also isolated GRP94 and GFP shRNA gene-transduced HeLa cells by infection with Ret/shGRP94 and Ret/GFP, respectively, for comparison. After removal of the uninfected and infected but inactivated fraction of cells by puromycin selection, the presence of retroviral DNA within the cellular genome was confirmed by PCR (data not shown). Total cell lysates were prepared from polyclonal pools of the transduced cells, and the expression of GRP78 protein was determined by Western blot analysis 
A

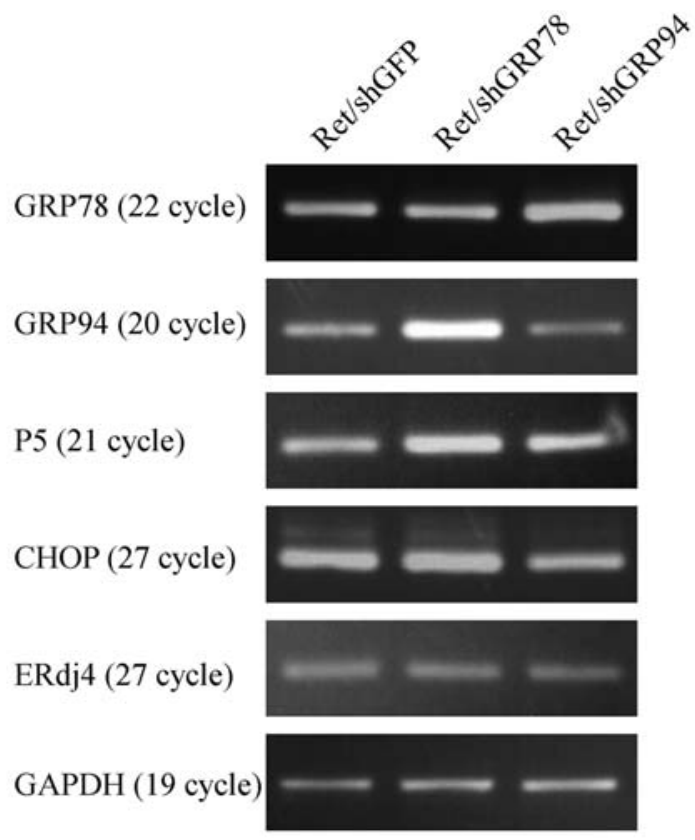

B

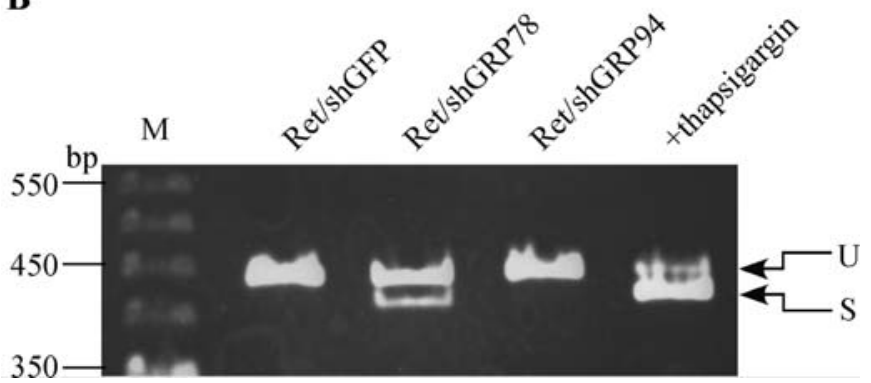

Figure 2. mRNA expression of GRP78, GRP94 and UPR-induced genes in Ret/shGRP78-, Ret/shGRP94- and control Ret/shGFP-transduced HeLa cells. (A) The mRNA levels of GRP78, GRP94, P5, CHOP and ERdj4 were analyzed by RT-PCR. The PCR cycles indicated in brackets were predetermined to allow semiquantitative comparisons among cDNAs developed from identical reverse transcriptase reactions. The reference gene, GAPDH, was amplified as an internal control. (B) Stable expression of GRP78 shRNA partially activates the Ire $1 \mathrm{p}-\mathrm{XBP} 1$ pathway. RT-PCR analysis of XBP1 mRNA was performed as previously described. The PCR products were separated on a $4 \%$ polyacrylamide gel and visualized by ethidium bromide staining. The positions of the unspliced (442 bp) and spliced (416 bp) products are shown as $\mathrm{U}$ and $\mathrm{S}$, respectively. RNA samples isolated from thapsigargin-treated $(1 \mu \mathrm{M}$ for $8 \mathrm{~h})$ cells were used as a positive control for activation of the Ire1p-XBP1 pathway.

(Fig. 1B). Contrary to our expectations, the amount of GRP78 protein was not reduced, but rather appeared to have slightly increased in Ret/shGRP78-transduced cells. Additionally, the amount of GRP94 had increased in the Ret/shGRP78transduced cells. On the other hand, a marked knockdown of GRP94 protein was observed in Ret/shGRP94-transduced cells, although the expression of the GRP78 protein appeared to have increased.

To determine whether the GRP78 shRNA can inhibit the expression of the target protein, the gene cassette was inserted into an adenovirus vector and used for transient expression of GRP78 shRNA. Adenovirus vectors containing GRP94 or the GFP shRNA gene were also used as controls.
A

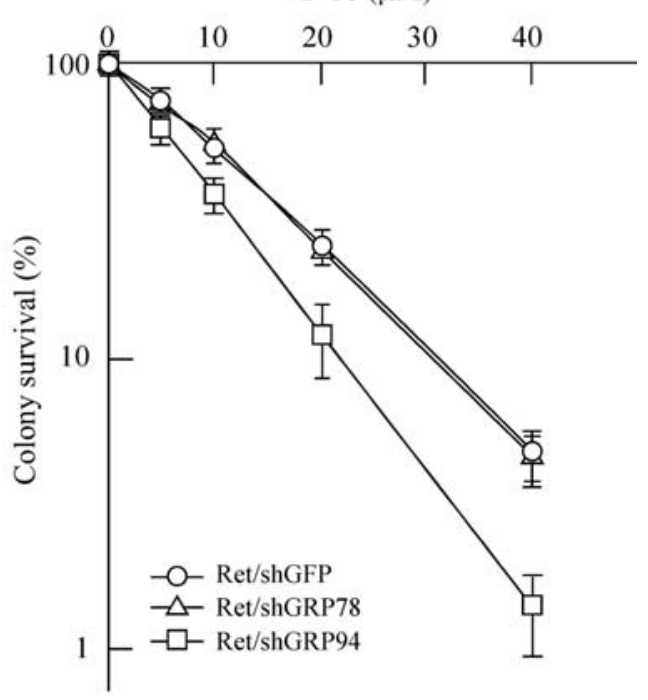

B

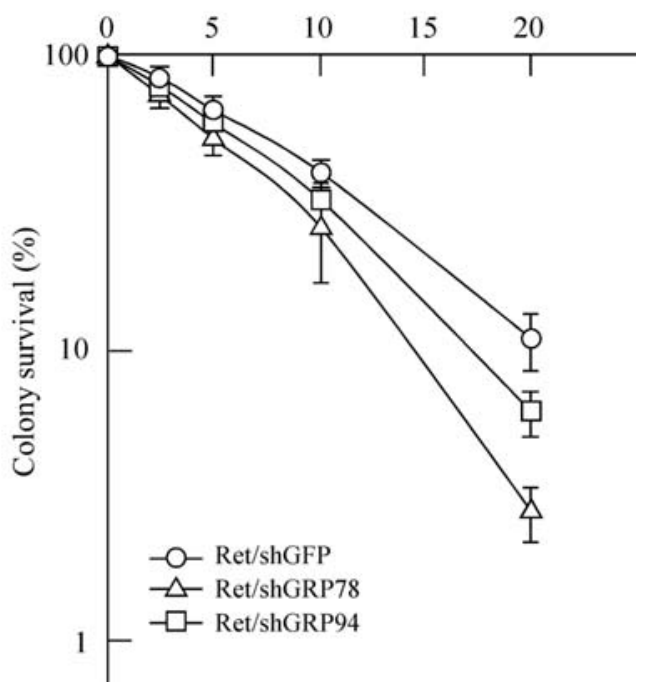

Figure 3. Comparison of sensitivity to VP-16 (A) and cisplatin (B) in Ret/shGRP78-, Ret/shGRP94- and control Ret/shGFP-transduced HeLa cells by a colony survival assay. Cells were exposed to VP-16 or cisplatin for $1 \mathrm{~h}$ at the different concentrations indicated. Values represent the percentage of colony numbers relative to those of mock-treated cells. Values are the mean \pm SD of three independent experiments.

HeLa cells express high levels of coxsackie-adenovirusreceptor and showed highly-efficient adenovirus-vectormediated transient transgene expression $(30,31)$. Two days after infection with each shRNA-expressing adenovirus vector at a MOI of 50, the expression of the targeted protein had decreased in both Ad/shGRP78- and Ad/shGRP94infected cells, as expected (Fig. 1C). This result demonstrates that the GRP78 shRNA designed by us can actually inhibit expression of the target protein when transiently introduced. The expression of the GRP94 protein also increased with Ad/shGRP78 infection, similar to when the cells were transfected with GRP78 siRNA (26). These results suggest that stable transduction of the GRP78 shRNA gene with the retrovirus vector is not capable of suppressing GRP78 


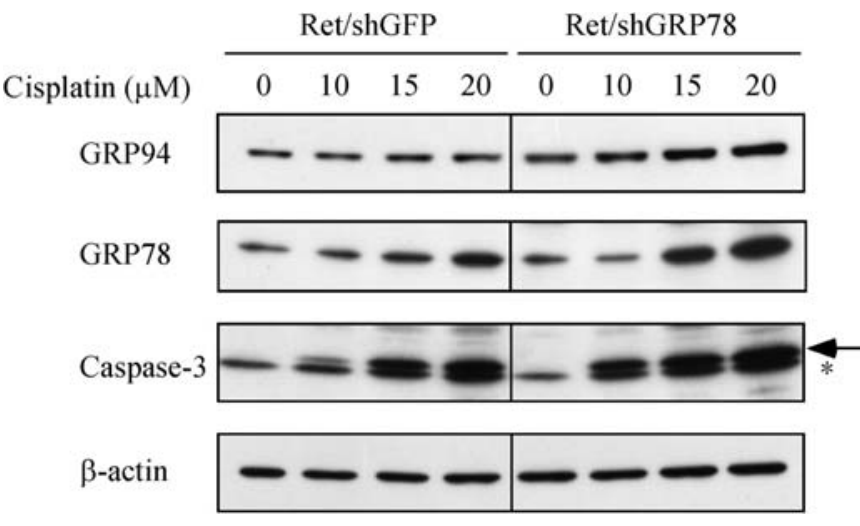

Figure 4. Activation of caspase-3 by cisplatin is enhanced in Ret/shGRP78 transduced HeLa cells. Cells were exposed to cisplatin for $1 \mathrm{~h}$ at the different concentrations indicated, cultured for a further $23 \mathrm{~h}$ in normal culture media, and then analyzed by immunoblotting using anti-GRP94 anti-GRP78, anti-cleaved form of caspase-3 and anti-actin antibodies. The amount of actin was used as a loading control. Bands representing the cleaved form of caspase- 3 are indicated by an arrow. The asterisk indicates non-specific bands.

expression in HeLa cells, even if the gene can suppress the expression of the target protein when it is transiently introduced.

Expression of UPR-induced genes increases in GRP78 shRNA gene-transduced HeLa cells. One possible explanation for the lack of suppression of GRP78 in Ret/shGRP78-transduced cells may be the activation of the UPR, as upregulation of UPR-induced genes and activation of UPR-dependent transcription was observed when HeLa cells were transfected with a GRP78 siRNA (26). Next, we analyzed the expression of three representative UPR-induced mRNAs, in addition to GRP78 and GRP94, by RT-PCR analysis in Ret/shGRP78transduced cells (Fig. 2A). The levels of GRP94 and P5 mRNA increased more than two-fold, whereas the levels of $C H O P$ and ERdj4 mRNA did not increase significantly. Reduction of GRP78 mRNA expression was not observed in Ret/shGRP78transduced cells. The mRNA levels of P5, CHOP and ERdj4 did not increase in Ret/shGRP94-transduced cells.

We previously reported that the spliced form of $X B P I$ mRNA, which increased after Ire $1 p$ activation and produces the active form of the XBP1 protein necessary for the induction of UPR-induced genes, increased in GRP78 siRNA-transfected HeLa cells (26). To ascertain that this pathway is also activated in Ret/shGRP78-transduced cells, we analyzed the amount of the spliced form of XBP1 mRNA by RT-PCR (Fig. 2B). As a positive control for UPR activation, thapsigargin-treated HeLa cells were also used. The amount of spliced mRNA (Fig. 2B) increased in Ret/shGRP78-transduced cells, although the extent of the increase was smaller than in thapsigargintreated cells.

These results suggest that the UPR is somewhat activated and that UPR-induced genes, including GRP78 itself, are moderately upregulated in Ret/shGRP78-transduced cells. It should be noted that the increased expression of GRP78 mRNA, as well as GRP78 protein, was observed in Ret/ shGRP94-transduced cells (Fig. 1B and 2A). This increase by the suppression of GRP94 may occur independently of UPR activation, as expression of other UPR-induced genes was not upregulated and the spliced form of XBPI mRNA did not increase in the Ret/shGRP94-transduced cells.

Sensitization to cisplatin by transduction of the GRP78 shRNA gene without suppression of GRP78 expression. Prolonged GRP78 shRNA expression could not maintain GRP78 knockdown because the levels of GRP78 protein in Ret/ shGRP78-transduced cells was similar to that in control Ret/shGFP-transduced cells. It is possible that continuous treatment for suppression of GRP78 function may activate the UPR and result in an adverse overexpression of GRP78, allowing the treated cancer cells to survive and maintain ERhomeostasis. Such an adaptation to suppression might also contribute to the resistance of cells to chemotherapy and radiotherapy.

To determine the effect of UPR activation on Ret/ shGRP78-transduced cells and their sensitivity to anticancer drugs, we treated the cells with various concentrations of VP-16 and cisplatin, which are clinically and widely used as anticancer drugs and whose sensitivities were reported to depend on the expression levels of GRP78 and GRP94 $(13,19,22,23)$. Sensitivity to VP-16 was similar between Ret/ shGRP78- and control Ret/shGFP-transduced cells (Fig. 3A). However, surprisingly, sensitivity to cisplatin was higher in Ret/shGRP78-transduced cells than in Ret/shGFP-transduced cells regardless of similar GRP78 protein levels in both cells (Fig. 3B). Colony survival data for Ret/shGRP94-transduced cells confirmed that knockdown of GRP94 increased sensitivity to VP-16 as previously reported (Fig. 3A) (18). Knockdown of GRP94 also sensitized HeLa cells to cisplatin although to a lesser extent than with GRP78 shRNA gene transduction (Fig. 3B).

To further confirm cisplatin sensitization by GRP78 shRNA expression without GRP78 knockdown, the amount of the active form of caspase- 3 was analyzed by Western blot analysis (Fig. 4). Compared with Ret/shGFP-transduced cells, the levels of active caspase-3 were higher in Ret/ shGRP78-transduced cells when treated with various concentrations of cisplatin for $24 \mathrm{~h}$. In addition, induction of both GRP78 and GRP94 by cisplatin was higher in Ret/shGRP78-transduced cells than in control Ret/shGFP cells, implying increased sensitivity to cisplatin in the Ret/shGRP78-transduced cells. These results suggest that GRP78 shRNA expression sensitized HeLa cells to cisplatin not via suppression of GRP78 but through mild activation of the UPR.

\section{Discussion}

A number of studies have reported that the downregulation of GRP78 expression with siRNA or antisense DNA sensitized cancer cells to chemotherapeutic agents, including VP-16 and cisplatin $(13,19,22,23)$. The reports suggested that GRP78 itself contributed to the resistance to those agents and could be a potential target for enhancing chemosensitivity of cancer cells. However, we previously reported that knockdown of GRP78 activated the UPR (26). Since the UPR is involved not only in apoptosis but also cell survival (6), the possibility that GRP78-targeted anticancer therapies might 
induce unfavorable events cannot be excluded, especially if the treatment is performed continuously or repeatedly.

In this study, we stably transduced HeLa cells with a GRP78 shRNA-expressing gene and found that this continuous approach for GRP78 knockdown was not effective, even if the same GRP78 shRNA gene can actually reduce the expression of GRP78 when transiently transduced with an adenovirus vector system (Fig. 1). Moreover, the expression of GRP94 and P5 mRNAs increased and was accompanied by UPR activation in the Ret/shGRP78transduced cells (Fig. 2). The reason why the expression of GRP78 did not decrease in the transduced cells is unclear. One possible explanation is that only the population of cells that were adapted for cell growth remained after puromycin selection, while the cells with suppressed GRP78 expression died due to strong UPR activation. This outcome is similar to what is observed in cells transfected with GRP78 siRNA (26). If so, the approach for GRP78 knockdown or inactivation should not be used continuously or repeatedly because it may no longer sensitize the cells to anticancer drugs. Indeed, sensitivity to VP-16-induced cell death in Ret/shGRP78transduced cells was similar to that in control Ret/shGFPtransduced cells (Fig. 3A).

Nevertheless, sensitivity to cisplatin was still higher in Ret/shGRP78-transduced cells when compared with control Ret/shGFP-transduced cells (Figs. 3B and 4). This could be because suppression of GRP78 expression is not involved in the sensitization to cisplatin by shRNA expression or siRNA treatment targeted to GRP78, but activation of the UPR is involved. Disruption of GRP78 metabolism by the expression of GRP78 shRNA may be involved in the activation of the UPR in Ret/shGRP78-transduced cells, although the apparent protein levels of GRP78 in the transduced cells were similar to those in control Ret/shGFP-transduced cells. This idea is further supported by several studies that reported higher sensitivity to cisplatin-induced cell death following upregulation of GRP78 with 2-deoxyglucose or 6-aminonicotineamide treatment in human colon cancer cells, human ovarian cancer cells and Chinese hamster V79 cells (32-34). In contrast, induction of ER chaperone proteins, including GRP78, by treatment with 2-deoxyglucose or A23817 conferred resistance of V79 and SK-MES-1 human lung cancer cells to doxorubicin, vincristine and VP-16 (35-37).

In addition, celecoxib, a non-steroidal anti-inflammatory drug that activates the UPR, potentiated the anticancer effect of cisplatin in vulvar cancer cells independently of cyclooxygenase $(38,39)$. Considering all of the various published studies, the possibility that the continuous but mild activation of the UPR by the expression of GRP78 shRNA can sensitize cancer cells to cisplatin is conceivable. Cisplatin has the unique ability to activate apoptosis via both DNA damage-dependent and -independent pathways, and the ER is likely its nonnuclear target (40). Induction of apoptosis by VP-16 requires nuclear events; cisplatin induced the activation of caspase-3 in enucleated cells (cytoplasts), but VP-16 did not (40). Therefore, the ability to activate two pathways for apoptosis may be involved in sensitization to cisplatin when the UPR is activated.

In summary, retroviral-mediated stable transduction of a GRP78 shRNA gene failed to suppress the expression of
GRP78 in HeLa cells. Upregulation of GRP94 and P5 mRNAs with a mild activation of UPR was observed in the transduced cells. However, sensitivity to cisplatin-induced cell death was higher in Ret/shGRP78-transduced cells than in control cells, suggesting that the activation of UPR is involved in sensitization. These results may offer a new chemotherapeutic approach using cisplatin.

\section{Acknowledgements}

This study was supported in part by Grants-in-Aid for Scientific Research through the Industrial Technology Research Grant Program in '04 from the New Energy and Industrial Technology Development Organization, the Smoking Research Foundation, the Tokyu Foundation for a Better Environment, the Tsuchiya Foundation, the Kieikai Research Foundation, and the Japan Society for the Promotion of Science. J. Lu was a doctoral student supported financially by an Ishidu Shun Memorial Scholarship and a Rotary Yoneyama Doctor Course Scholarship. G. Hu is a doctoral student supported financially by a MEXT Honors Scholarship for Privately Financed International Students.

\section{References}

1. Brown JM and Giaccia AJ: The unique physiology of solid tumors: opportunities (and problems) for cancer therapy. Cancer Res 58: 1408-1416, 1998

2. Kaufman RJ, Scheuner D, Schroder M, et al: The unfolded protein response in nutrient sensing and differentiation. Nat Rev Mol Cell Biol 3: 411-421, 2002.

3. Wouters BG and Koritzinsky M: Hypoxia signalling through mTOR and the unfolded protein response in cancer. Nat Rev Cancer 8: 851-864, 2008.

4. Lee AS: The glucose-regulated proteins: stress induction and clinical applications. Trends Biochem Sci 26: 504-510, 2001.

5. Lee AS: GRP78 induction in cancer: therapeutic and prognostic implications. Cancer Res 67: 3496-3499, 2007.

6. Healy SJ, Gorman AM, Mousavi-Shafaei P, Gupta S and Samali A: Targeting the endoplasmic reticulum-stress response as an anticancer strategy. Eur J Pharmacol 625: 234-246, 2009.

7. Daneshmand S, Quek ML, Lin E, et al: Glucose-regulated protein GRP78 is up-regulated in prostate cancer and correlates with recurrence and survival. Hum Pathol 38: 1547-1552, 2007.

8. Fernandez PM, Tabbara SO, Jacobs LK, et al: Overexpression of the glucose-regulated stress gene GRP78 in malignant but not benign human breast lesions. Breast Cancer Res Treat 59: 15-26, 2000 .

9. Wang Q, He Z, Zhang J, et al: Overexpression of endoplasmic reticulum molecular chaperone GRP94 and GRP78 in human lung cancer tissues and its significance. Cancer Detect Prev 29: 544-551, 2005.

10. Langer R, Feith M, Siewert JR, Wester HJ and Hoefler H: Expression and clinical significance of glucose regulated proteins GRP78 (BiP) and GRP94 (GP96) in human adenocarcinomas of the esophagus. BMC Cancer 8: 70, 2008.

11. Zheng HC, Takahashi H, Li XH, et al: Overexpression of GRP78 and GRP94 are markers for aggressive behavior and poor prognosis in gastric carcinomas. Hum Pathol 39: 1042-1049, 2008.

12. Xing X, Lai M, Wang Y, Xu E and Huang Q: Overexpression of glucose-regulated protein 78 in colon cancer. Clin Chim Acta 364: 308-315, 2006.

13. Lee HK, Xiang C, Cazacu S, et al: GRP78 is overexpressed in glioblastomas and regulates glioma cell growth and apoptosis. Neuro Oncol 10: 236-243, 2008.

14. Shuda M, Kondoh N, Imazeki N, et al: Activation of the ATF6, $\mathrm{XBP} 1$ and grp78 genes in human hepatocellular carcinoma: a possible involvement of the ER stress pathway in hepatocarcinogenesis. J Hepatol 38: 605-614, 2003.

15. Kleizen B and Braakman I: Protein folding and quality control in the endoplasmic reticulum. Curr Opin Cell Biol 16: 343-349, 2004. 
16. Yang $\mathrm{Y}$ and Li Z: Roles of heat shock protein gp96 in the ER quality control: redundant or unique function? Mol Cells 20: 173-182, 2005.

17. $\mathrm{Fu} \mathrm{Y}$ and Lee $\mathrm{AS}$ : Glucose regulated proteins in cancer progression, drug resistance and immunotherapy. Cancer Biol Ther 5: 741-744, 2006.

18. Reddy RK, Lu J and Lee AS: The endoplasmic reticulum chaperone glycoprotein GRP94 with Ca(2+)-binding and antiapoptotic properties is a novel proteolytic target of calpain during etoposide-induced apoptosis. J Biol Chem 274: 28476-28483, 1999.

19. Li J and Lee AS: Stress induction of GRP78/BiP and its role in cancer. Curr Mol Med 6: 45-54, 2006.

20. Kubota H, Suzuki T, Lu J, et al: Increased expression of GRP94 protein is associated with decreased sensitivity to X-rays in cervical cancer cell lines. Int J Radiat Biol 81: 701-709, 2005.

21. Zhang LY, Zhang XC, Wang LD, Zhang ZF and Li PL: Increased expression of GRP94 protein is associated with decreased sensitivity to adriamycin in ovarian carcinoma cell lines. Clin Exp Obstet Gynecol 35: 257-263, 2008.

22. Jiang CC, Mao ZG, Avery-Kiejda KA, Wade M, Hersey P and Zhang XD: Glucose-regulated protein 78 antagonizes cisplatin and adriamycin in human melanoma cells. Carcinogenesis 30 : 197-204, 2009.

23. Wang Y, Wang W, Wang S, Wang J, Shao S and Wang Q Down-regulation of GRP78 is associated with the sensitivity of chemotherapy to VP-16 in small cell lung cancer NCI-H446 cells. BMC Cancer 8: 372, 2008

24. Dong D, Ni M, Li J, et al: Critical role of the stress chaperone GRP78/BiP in tumor proliferation, survival, and tumor angiogenesis in transgene-induced mammary tumor development. Cancer Res 68: 498-505, 2008

25. Zhang K and Kaufman RJ: Signaling the unfolded protein response from the endoplasmic reticulum. J Biol Chem 279: 25935-25938, 2004

26. Suzuki T, Lu J, Zahed M, Kita K and Suzuki N: Reduction of GRP78 expression with siRNA activates unfolded protein response leading to apoptosis in HeLa cells. Arch Biochem Biophys 468: 1-14, 2007.

27. Brummelkamp TR, Bernards R and Agami R: Stable suppression of tumorigenicity by virus-mediated RNA interference. Cancer Cell 2: 243-247, 2002.

28. Brummelkamp TR, Bernards R and Agami R: A system for stable expression of short interfering RNAs in mammalian cells Science 296: 550-553, 2002.
29. Deregowski V and Canalis E: Gene delivery by retroviruses. Methods Mol Biol 455: 157-162, 2008.

30. Fechner H, Wang X, Wang H, et al: Trans-complementation of vector replication versus Coxsackie-adenovirus-receptor overexpression to improve transgene expression in poorly permissive cancer cells. Gene Ther 7: 1954-1968, 2000.

31. Gurzov EN and Izquierdo M: RNA interference against Hec1 inhibits tumor growth in vivo. Gene Ther 13: 1-7, 2006.

32. Belfi CA, Chatterjee S, Gosky DM, Berger SJ and Berger NA: Increased sensitivity of human colon cancer cells to DNA crosslinking agents after GRP78 up-regulation. Biochem Biophys Res Commun 257: 361-368, 1999.

33. Chatterjee S, Hirota H, Belfi CA, Berger SJ and Berger NA: Hypersensitivity to DNA cross-linking agents associated with up-regulation of glucose-regulated stress protein GRP78. Cancer Res 57: 5112-5116, 1997.

34. Yamada M, Tomida A, Yun J, et al: Cellular sensitization to cisplatin and carboplatin with decreased removal of platinumDNA adduct by glucose-regulated stress. Cancer Chemother Pharmacol 44: 59-64, 1999.

35. Hughes CS, Shen JW and Subjeck JR: Resistance to etoposide induced by three glucose-regulated stresses in Chinese hamster ovary cells. Cancer Res 49: 4452-4454, 1989.

36. Shen J, Hughes C, Chao C, et al: Coinduction of glucoseregulated proteins and doxorubicin resistance in Chinese hamster cells. Proc Natl Acad Sci USA 84: 3278-3282, 1987.

37. Zhang L, Wang S, Wangtao, et al: Upregulation of GRP78 and GRP94 and its function in chemotherapy resistance to VP-16 in human lung cancer cell line SK-MES-1. Cancer Invest 27: 453-458, 2009.

38. Kim SH, Song YC and Song YS: Celecoxib potentiates the anticancer effect of cisplatin on vulvar cancer cells independently of cyclooxygenase. Ann NY Acad Sci 1171: 635-641, 2009.

39. Tsutsumi S, Namba T, Tanaka KI, et al: Celecoxib upregulates endoplasmic reticulum chaperones that inhibit celecoxib-induced apoptosis in human gastric cells. Oncogene 25: 1018-1029, 2006.

40. Mandic A, Hansson J, Linder S and Shoshan MC: Cisplatin induces endoplasmic reticulum stress and nucleus-independent apoptotic signaling. J Biol Chem 278: 9100-9106, 2003. 